\title{
Genetic alterations and pathways in patients with Hereditary Angioedema of Unknown Cause (U-HAE)
}

\author{
Hande KAYMAKCALAN ${ }^{1}$ (D), Hande ALP ${ }^{2}$ (D), Ahmet Okay CAGLAYAN ${ }^{3}$ (D), Okan GULBAHAR ${ }^{4}$, Emine Nihal GOKMEN (D), $^{\circ}$ \\ Emrah NIKEREL ${ }^{5}$ (D) \\ ${ }^{1}$ Department of Pediatrics, School of Medicine, Demiroglu Science University, Istanbul, Turkey. \\ ${ }^{2}$ Pediatrics, Private Clinic, Ankara, Turkey. \\ ${ }^{3}$ Department of Medical Genetics, School of Medicine, 9 Eylul University, Izmir, Turkey. \\ ${ }^{4}$ Department of Allergy and Immunology, School of Medicine, Ege University, Izmir, Turkey. \\ ${ }^{5}$ Department of Genetics and Bioengineering, Faculty of Engineering, Yeditepe University, Istanbul, Turkey.
}

Corresponding Author: Hande KAYMAKCALAN

E-mail: doctorhande@yahoo.com

Submitted: 12.03.2021 Accepted: 07.06.2021

\section{ABSTRACT}

Objective: Hereditary angioedema ( HAE) with normal C1 inhibitor (HAE-nC1-INH), is a genetically complex, rare disease and mutations in F12, ANGPT1, PLG, MYOF genes are found in some families with HAE-nC1-INH. However, often a specific mutation cannot be identified and this type is called as hereditary angioedema of unknown cause (U-HAE). Our aim was to identify putative causative genetic alterations and/or pathways by whole exome sequencing in patients with U-HAE.

Patients and Methods: Nine patients from 8 families between the ages of 3 to 63 years with U-HAE and 6 controls were enrolled for the study and whole exome sequencing were performed.

Results: No significant difference was found between the case and control group for the a priori suspected set of genes. Variants in the genes; RAMP2, IL6, GP1BA, C1QBP were significantly different between U-HAE and control group. Downstream functional analysis found that blood coagulation pathways were enriched in these genes.

Conclusion: Proteins that are not involved in contact pathways may also play a role in U-HAE. These variants need to be replicated in larger cohorts and studied at the functional level to verify our findings.

Keywords: Hereditary angioedema of unknown cause ( U-HAE), Whole exome sequencing (WES ), Genetic

\section{INTRODUCTION}

Hereditary angioedema (HAE) is a rare autosomal dominant disease that results in recurrent attacks of swelling and has a highly variable clinical course [1]. There are two well defined types of HAE: HAE due to $\mathrm{C} 1$ inhibitor deficiency $(\mathrm{C} 1-\mathrm{INH})$ and HAE with normal C1-INH deficiency (HAE-nlC1-INH). HAE due to $\mathrm{C} 1-\mathrm{INH}$ deficiency is further subdivided into type 1 (low plasma c1INH antigen) and type 2 (normal, but dysfunctional C1INH). Both HAE types are caused by mutations in SERPING1 gene $[2,3]$.

Hereditary angioedema with nlC1-INH is a genetically complex, rare disease first described in 2000. Prevalence is estimated at 1:100.000. Mutations in F12 gene are seen in approximately
$30 \%$ of the patients and recently mutations in ANGPT1, PLG and MYOF were shown in some families with HAE-nC1-INH [4] .However, most of the time a specific mutation cannot be shown. There is a need for methods that can identify affected individuals with HAE-nC1-INH with unknown genetic cause (U-HAE) $[5,1]$.

Functional genomics approaches attempt to discover, define and describe function of genes and interactions thereof, making use of the vast data generated by genomic and transcriptomic projects [6]. One initial step into this endeavor, is the discovery of causative genes, in particular for a disease or trait of interest

How to cite this article: Kaymakcalan H, Alp H, Caglayan AO,Gulbahar O, Gokmen EN, Nikerel E. Genetic alterations and pathways in patients with hereditary angioedema of unknown cause. Marmara Med J 2021; 34(3):274-278. doi: 10.5472/marumj.1009115 
via observational studies, where features (e.g. structural variants in the genome) are associated with the traits.

\section{PATIENTS and METHODS}

After ethics approval (Ankara University Ethics Committee, date: 08/27/2012 number: 13-419-12) and consent forms were taken, 9 patients between the ages of 3 to 63 years with U-HAE were enrolled in the study ( Table I). 8 patients were from a Turkish decent and 1 was Irish-American. 2 patients were father and his adult daughter, and the rest belonged to unrelated families. 3 of the index cases were younger than 11 years of age. 6 controls were taken randomly from a sample of patients with congenital heart disease. All index cases presented with recurrent angioedema symptoms and met the criteria for HAEnlC1-INH as described by Cicardi et al [1].

\section{WES analysis, Identification of variants and statistical procedures, bioinformatics analyses.}

All patients (controls and cases) underwent to whole-exome sequencing (WES) analysis. The raw sequence files were quality filtered (average Phred score $>30$, minimum 50X depth of coverage) using FastQC. The resulting sequence files were mapped to human genome (hg19) using Bowtie2 [7] or BurrowsWheeler Alignment (BWA) tool [8] and downstream analyses were performed using Sam tools [9]. The mapped reads were further used for variant detection using standard bioinformatics workflows of samtools and/or GATK [10]. All detected variants were listed and inspected for their PolyPhen [11] and SIFT [12] scores and further checked in COSMIC database [13]. The variations identified as "benign" were discarded and variations with outcomes as "possibly damaging" and "unknown" were kept.

\section{Construction of Variant profiles per gene}

To assess potential association between the U-HAE and structural variants, variant profile for each gene is constructed as follows: for each gene and each sample considered, a binary variable that represents the presence or absence of a structural variation (with suitable PolyPhen outcome) is generated. In doing so, all variations in the selected gene are aggregated. In case there are multiple variations in a gene, the binary variable is still taken as one. This yields variant profiles per gene for case and control groups. The resulting contingency table, taking cases and controls as well as number of variations and non-variations is tested using Fisher's exact test [14] for the null hypothesis that there is no difference in variation profile between the case and control group. $\alpha=0.05$ is taken as the significance threshold.

\section{Identification of potential candidate genes}

Initial gene set, for which a variation profile is generated and tested for significance among groups is constructed based on reported genes for HAE variants and literature search. To expand and identify further potential genes, we used the initial gene set consisting of F12, ACE, ADM and SERPINA1 as a starting point, and considered genes interacting at protein level (proteinprotein interactions) to potentially associate with U-HAE case.
The protein-protein interactions are retrieved from STRING database [15] .

\section{Downstream Bioinformatics analyses}

The final gene list containing the genes with statistically significant difference in their variation profile between the case and control is further functionally analyzed for their function and GO-terms using The Database for Annotation, Visualization and Integrated Discovery (DAVID) database [16,17] and amiGO tool [18].

\section{RESULTS}

\section{Characteristics of the Patients}

In this study, 9 patients from 8 families and 6 controls with different clinical profile were analyzed. Characteristics of the patients are shown in Table I.

Table I. Characteristics of patients with U-HAE
\begin{tabular}{|lccl|}
\hline Patient & Sex & Age at diagnosis & Presenting Symptoms \\
\hline 1 & M & 33 & Lip swelling \\
\hline 2 & F & 9 & Lip and hands swelling \\
\hline 3 & M & 18 & Lip and scrotum swelling \\
\hline 4 & F & 22 & Tongue and lip swelling \\
\hline 5 & F & 34 & Tongue swelling \\
\hline 6 & F & 7 & Lip swelling \\
\hline 7 & M & 50 & Tongue swelling, abdominal pain \\
\hline 8 & & & $\begin{array}{l}\text { Acute abdominal pain with free fluid } \\
\text { collection }\end{array}$ \\
\hline 9 & F & 19 & Hands swelling \\
\hline
\end{tabular}

\section{Analysis of Variant profiles for F12, ACE, SERPINA1 and ADM}

In the first round the initial seed gene list consisting of F12, ACE, SERPINA1 and ADM are inspected for their variant profiles, different from case to control.

In an attempt to characterize correctly the HAE and making sure that these patients are U-HAE, an additional list of genes was also inspected for their variant profile. This list is composed of genes that are used to characterize the HAE in earlier works. The results, including the seed gene list as well as candidate genes, are given in Figure 1. Only high-quality variants were included in the analysis with possibly damaging and unknown variants based on their polyphen scores. The analysis resulted in no gene with a differential variant profile among case and control groups. Interestingly, no variation was observed for SERPINA1 and ADM genes in the cohort and almost all individuals including control had variation for $\mathrm{F} 12(\mathrm{p}=0.476)$ and ACE $(\mathrm{p}=0.999)$. The lowest among those was the variant profile of SERPING1 gene which was only marginally significant $(\mathrm{p}=0.044)$ ( Figure 1 ).

\section{Expanding the search space using interactome information}

In searching for causative genes in this dataset, we expanded the initial gene set by including the genes whose protein products 
were reported to be interacting with the initial set, based on STRING database and selected only the high-confidence pairs (minimum required interaction score $>0.9$, Figure 2). The new gene list contained 29 additional genes, for which variation profiles were constructed, tested for significant difference between the case and control groups and found that indeed significant difference was found for this set $(\mathrm{p}=0.027)$.

After seeing that not all genes in the expanded list were different in their variant profile, we focused on genes in the new list that would best differentiate (lowest p-value) the case and control groups, based on the variant profiles. To achieve this, each gene in the expanded list was ranked for their p-value and the final list of genes is given in Table II. Using these 4 genes, $\mathrm{p}$-value was estimated to be 0.023 .

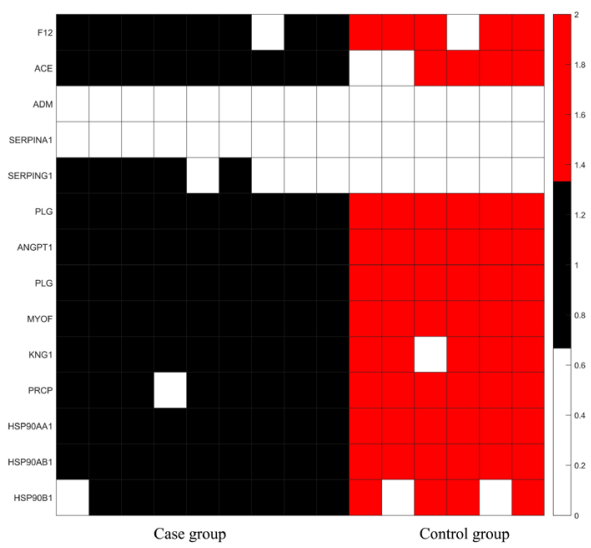

Figure 1. Variant profiles for possibly damaging and unknown variants for case (black) and control (red) groups. Colored spots present the presence of variant in a selected gene in the respective sample and white represents the absence. In this cohort, there are no possibly damaging and unknown variants for ADM and SERPINA1 genes.

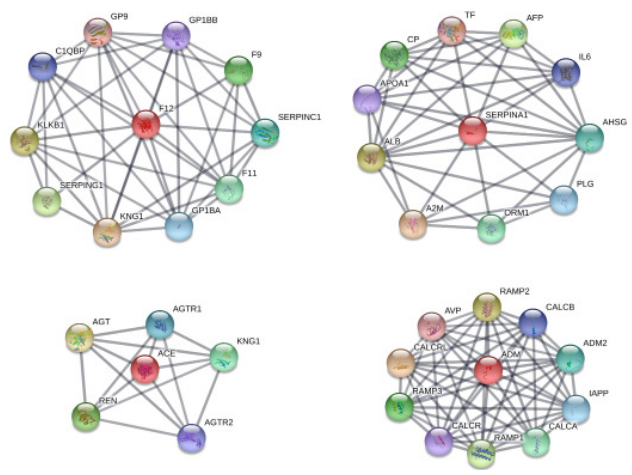

Figure 2. Searching for causative genes U-HAE. The expanded list of genes constructed by considering highest confidence interactions according to STRING database with the initial set of genes. Each network in the figure is centered around one of the genes in the initial set: F12, SERPINA1, ACE, ADM from left to right, top to bottom. The thickness of the lines connecting each protein couple indicates the strength of data support as provided by STRING database.
Table II. Final list of genes proposed in this study, based on statistical association of the variant profile with U-HAE diagnosis state and their variant profiles for case and control groups.

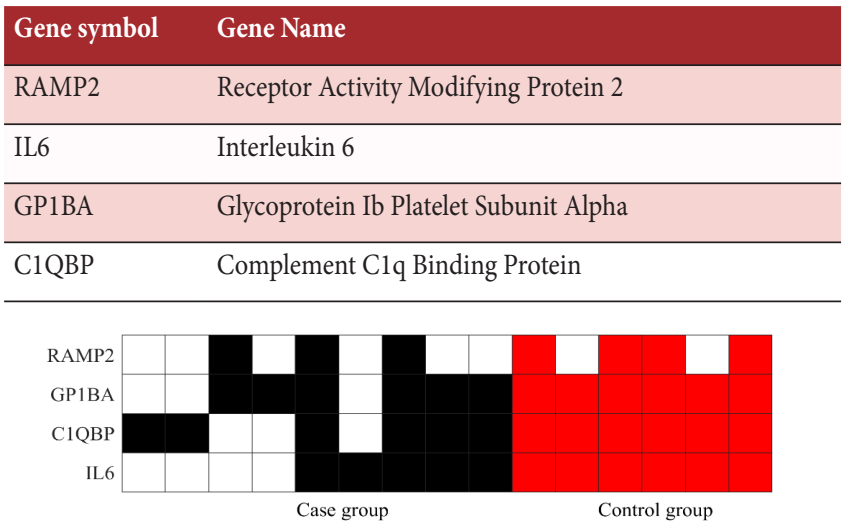

\section{Downstream analysis of the proposed genes}

The obtained set of genes were downstream analyzed to be inspected for their (common) function/pathway using GO Enrichment Analysis via amiGO tool. The GO terms overrepresented for the gene list in Table II is given in Table III.

Table III. GO terms overrepresented for the candidate genes in Table I.

\begin{tabular}{|l|c|}
\hline GO biological process & $\begin{array}{c}\text { FDR corrected } \\
\text { p value }\end{array}$ \\
\hline blood coagulation, intrinsic pathway (GO:0007597) & $3.76 \mathrm{E}-02$ \\
\hline blood coagulation, fibrin clot formation (GO:0072378) & $5.54 \mathrm{E}-02$ \\
\hline protein activation cascade (GO:0072376) & $4.15 \mathrm{E}-02$ \\
\hline blood coagulation (GO:0007596) & $2.95 \mathrm{E}-02$ \\
\hline coagulation (GO:0050817) & $2.58 \mathrm{E}-02$ \\
\hline hemostasis (GO:0007599) & $2.33 \mathrm{E}-02$ \\
\hline $\begin{array}{l}\text { negative regulation of response to external stimulus } \\
\text { (GO:0032102) }\end{array}$ & $8.75 \mathrm{E}-03$ \\
\hline regulation of response to external stimulus (GO:0032101) & $3.48 \mathrm{E}-02$ \\
\hline
\end{tabular}

FDR: False Discovery Rate, GO: Gene Ontology

\section{DISCUSSION}

We propose a data-mined set of genes that statistically show variation among U-HAE cases and control groups. Prominent among this set of genes are RAMP2, IL6, GP1BA and C1QBP. RAMP2 can function as an adrenomedullin receptor ( ADM) which is a vasoactive peptide and it has been shown that its level increases during C1-INH-HAE attacks together with other vasoactive peptides arginine vasopressin (AVP) and endothelin-1 (ET-1) to counter-balance the actions of excess bradykinin (BK) and terminate the attacks [19]. HAE-nlC1INH may also be BK mediated as they show a favorable response to $\mathrm{BK}$ pathway-targeted medications [20] .The possible effects of RAMP2 in vasoactive peptides may reveal a novel pathophysiological aspect. 
Concentrations of interleukin ( IL)-1, IL-6, and transforming growth factor (TGF- $\beta$ ) were found to be significantly higher in HAE patients in remission compared with healthy controls. [21]. IL-6 is a soluble mediator with a pleiotropic effect on inflammation, immune response, and hematopoiesis. IL-6 induces excess production of vascular endothelial growth factor (VEGF), leading to enhanced angiogenesis and increased vascular permeability which causes joint swelling and edema. VEGF also interacts with BK to mediate plasma leakage.

Glycoprotein Ibalpha ( GP1BA) is a platelet surface membrane glycoprotein that functions as a receptor for von Willebrand factor. The binding of VWF to the GP Ib complex initiates signaling events within the platelet that lead to enhanced platelet activation, thrombosis, and hemostasis [22]. Mutations in this gene is associated with Bernard-Soulier syndrome and platelet type Von-Willebrand Disease. This glycoprotein may play a role in U-HAE by platelet activation, but this is another research topic.

Complement component 1q $(C 1 Q B P)$ subcomponent binding protein is a multifunctional protein involved in immune response, energy homeostasis of cells as a plasma membrane receptor, and a nuclear, cytoplasmic or mitochondrial protein. It has a role in the mediation of the actions of pro-inflammatory agents, such as high molecular weight kininogen to produce further pro-inflammatory agents [23]. It can play as a mediator in U-HAE since it acts as a bridge between the complement and contact activation system.

.U-HAE is usually misdiagnosed or underdiagnosed due to rarity of the disease and lack of specific biomarkers. This results in mismanagement of this potentially life threatening disease.

Detection of causative genes will help in timely diagnosis of patients and identifying affected family members. It will also allow for understanding the pathophysiology of this disorder, which in turn will lead to better therapeutic options. In our study genes that statistically show variation among U-HAE cases and control group are RAMP2, IL6, GP1BA and C1QBP.

Our study contributes to understanding the potential genetic pathways in U-HAE. The next step would be to increase our sample size, do family segregation studies, to extend the work to GWAS or RNA-Seq studies to further our understanding via network analyses and functional (e.g., transcriptomic or low throughput) analyses to understand and confirm the disease modifying effects of these genetic variations.

\section{Compliance with Ethical Standards}

Ethical Approval: The study was approved by the Ankara University Ethics Committee (date: 08/27/2012 number: 13419-12) and consent forms were taken.

Financial Support: The authors have no relevant financial information to disclose.

Conflict of interest: The authors declare that they have no conflict of interest to declare

Authors' contribution: H.K.: conceived and designed the analysis, collected the data of the patients, did the literature search and wrote the article. H.A.: conceived and designed the analysis, collected the data of the patients, did the literature search. A.O.C.: performed the genetic analyses. O.G.: collected the data of the patients, did the literature search, contributed to the conception of the research. E.N.G.: collected the data of the patients, contributed to the conception of the research. E.N.: did the statistical analysis and literature search, contributed to the conception of the research, wrote the article. All authors discussed the results and approved the final version of the article.

\section{REFERENCES}

[1] Cicardi M, Aberer W, Banerji A et al. HAWK under the patronage of EAACI (European Academy of Allergy and Clinical Immunology). Classification, diagnosis, and approach to treatment for angioedema: consensus report from the Hereditary Angioedema International Working Group. Allergy 2014;69:602-16. doi: 10.1111/all.12380.

[2] Bork K, Barnstedt SE, Koch P, Traupe H. Hereditary angioedema with normal C1-inhibitor activity in women. Lancet 2000;356(9225):213-7. doi: 10.1016/S01406736(00)02483-1.

[3] Dewald G, Bork K. Missense mutations in the coagulation factor XII (Hageman factor) gene in hereditary angioedema with normal C1 inhibitor. Biochem Biophys Res Commun 2006 May 19;343(4):1286-9. doi: 10.1016/j.bbrc.2006.03.092.

[4] Cichon S, Martin L, Hennies HC et al. Increased activity of coagulation factor XII (Hageman factor) causes hereditary angioedema type III. Am J Hum Genet 2006 Dec;79(6):1098104. doi: 10.1086/509899.

[5] Bodian DL, Vilboux T, Hauser NS. Genotype-first analysis of a generally healthy population cohort supports genetic testing for diagnosis of hereditary angioedema of unknown cause. Allergy Asthma Clin Immunol 2019; 16:15:32. doi: 10.1186/ s13223.019.0346-1.

[6] Hieter P, Boguski M. Functional genomics: it's all how you read it. Science 1997; 24:278(5338):601-2. doi: 10.1126/ science.278.5338.601.

[7] Langmead B, Salzberg SL. Fast gapped-read alignment with Bowtie 2. Nat Methods 2012;9:357-9.

[8] Li H, Durbin R. Fast and accurate short read alignment with Burrows-Wheeler transform. Bioinformatics 2009; 25:175460. doi: 10.1093/bioinformatics/btp324

[9] Li H, Handsaker B, Wysoker A, et al. 1000 Genome Project Data Processing Subgroup. The Sequence Alignment/Map format and SAMtools. Bioinformatics 2009; 15:25:2078-9. doi: 10.1093/bioinformatics/btp352.

[10] Van der Auwera GA, Carneiro MO, Hartl C, et al. From FastQ data to high confidence variant calls: the Genome Analysis Toolkit best practices pipeline. Curr Protoc Bioinformatics 2013;43(1110):11.10.1-11.10.33. doi:10.1002/047.125.0953. bi1110s43. .

[11] Adzhubei IA, Schmidt S, Peshkin L, et al. A method and server for predicting damaging missense mutations. Nat Methods. 2010;7:248-9. doi: 10.1038/nmeth0410-248. 
[12] Sim NL, Kumar P, Hu J, et al. SIFT web server: predicting effects of amino acid substitutions on proteins. Nucleic Acids Res 2012;40 (Web Server issue):W452-7. doi: 10.1093/nar/ gks539

[13] Tate JG, Bamford S, Jubb H, et al. COSMIC: the Catalogue of Somatic Mutations in Cancer. Nucleic Acids Res. 2019;47(D1):D941-D947. doi: 10.1093/nar/gky1015.

[14] Wang MH, Cordell HJ, Van Steen K. Statistical methods for genome-wide association studies. Semin Cancer Biol 2019;55:53-60. doi: 10.1016/j.semcancer.2018.04.008.

[15] Szklarczyk D, Gable AL, Lyon D, et al. STRING v11: proteinprotein association networks with increased coverage, supporting functional discovery in genome-wide experimental datasets. Nucleic Acids Res 2019; 8;47(D1):D607-D613. doi: 10.1093/nar/gky1131.

[16] Huang da W, Sherman BT, Lempicki RA. Bioinformatics enrichment tools: paths toward the comprehensive functional analysis of large gene lists. Nucleic Acids Res 2009;37:1-13. doi: 10.1093/nar/gkn923.

[17] Huang da W, Sherman BT, Lempicki RA. Systematic and integrative analysis of large gene lists using DAVID bioinformatics resources. Nat Protoc 2009;4:44-57. doi: 10.1038/nprot.2008.211

[18] The Gene Ontology Consortium. The Gene Ontology Resource: 20 years and still GOing strong. Nucleic Acids Res 2019;47(D1):D330-D338. doi: 10.1093/nar/gky1055.

[19] Kajdácsi E, Jani PK, Csuka D, et al. J Clin Immunol 2016;36:160-70. doi: 10.1007/s10875.016.0239-8

[20] Cicardi M, Zuraw BL. Angioedema due to bradykinin dysregulation. J Allergy Clin Immunol Pract 2018;6:1132-41. doi: 10.1016/j.jaip.2018.04.022.

[21] Arcoleo F, Lo Pizzo M, Misiano G, et al. The complex alteration in the network of IL-17-type cytokines in patients with hereditary angioedema. Clin Exp Med. 2018;18:355-361. doi:10.1007/s10238.018.0499-022.

[22] López JA, Andrews RK, Afshar-Kharghan V, et al. BernardSoulier syndrome. Blood 1998;91:4397-418. doi: 10.1182/ blood.V91.12.4397

[23] Peerschke EI, Ghebrehiwet B. The contribution of gC1qR/p33 in infection and inflammation. Immunobiology 2007;212:33342. doi: 10.1016/j.imbio.2006.11.011. 